\title{
Efficacy of Sofosbuvir and Ledipasvir in Comparison to Sofosbuvir and Daclatasvir in Management of Egyptian Chronic Hepatitis C Patients \\ Nouh, Israa Alaa ${ }^{1}$, Mostafa, Adila ${ }^{2}$, Elsheimy, Eman², Sief, Sameh ${ }^{1}$ Eysa, Basem ${ }^{1}$, \\ ${ }^{1}$ Tropical Medicine Department, National Hepatology and Tropical Medicine Research Institute, Egypt. \\ ${ }^{2}$ Hepatology, Gastroenterology and Infectious Diseases Department ,Faculty of Medicine for Girls, AL-Azhar University, Egypt.
}

Corresponding Author Nouh, Israa Alaa

Mobile:

$+201224991600$

E mail: israa.nouh@gmail. com

Key words: DAAS, Daclatasvir, Ledipasvir \& SVR
Background and study aim: In Egypt, the prevalence of hepatitis $\mathrm{C}$ virus (HCV) antibodies is the highest worldwide by 7.6\%. Applying efficient treatment protocol on large scale could decrease $\mathrm{HCV}$ prevalence as well as disease burden.The aim of this study is to compare the efficacy of Sofosbuvir plus ledipasvir versus Sofosbuvir plus daclatasvir in management of chronic hepatitis C Egyptian patients with either easy to treat (naive patients with Child score A5)or difficult to treat (interferon experienced).

Patients and Methods: the study was performed on 200 chronic hepatitis C patients and they were classified into Group I: Included 100 patients fulfill criteria of [easy to treat] and were subdivided into two subgroups; Group Ia: 50 patients were treated with daily Sofosbuvir (400 mg) plus Daclatasvir (60 $\mathrm{mg}$ ) for 12 weeks. Group Ib: 50 patients were treated with daily Sofosbuvir (400 $\mathrm{mg}$ ) plus Ledipasvir (90 mg) for 12 weeks. Group II: Included 100 patients fulfill criteria of (difficult to treat) and were subdivided into two subgroups; Group IIa: 50 patients were treated with daily Sofosbuvir (400 $\mathrm{mg})$ and Daclatasvir $(60 \mathrm{mg})$ plus daily weightbased Ribavirin for 12 weeks; Group IIb:
50 patients were treated with daily Sofosbuvir (400 mg) ) plus Ledipasvir (90 mg) plus daily weight-based Ribavirin for 12 weeks.

Results: GIa: 50 patients treated with Sof-Dac showed $100 \%$ cure (-ve PCR) at wk12 and wk 24. GIb: 50 patients treated with Sof-Led showed 98\% cure (-ve PCR) at wk12 and wk 24. GIIa: 50 patients treated with Sof-Dac-Riba showed $100 \%$ cure (-ve PCR) at wk12 and $98 \%$ at wk 24. GIIb: 50 patients treated with SofLed-Riba showed $98 \%$ cure (-ve PCR) at wk12 and $96 \%$ at wk 24.The difference between treatment regimens (Sof + Dac or Sof + Lid with or without Riba), regarding the cure rate and adverse events in all the studied groups was not significant which indicates the safety and effectiveness of both medication regimens. The decrease in FIB4 calculation at week 24 was highly significant in comparison to the baseline in GIa (2.23 to 0.67) and GIb (2.1 to 1.12) (easy to treat, while the difference in FIB4 calculation was not statistically significant at week 24 in GIIa and GIIb (difficult to treat).

Conclusion: Both regimens are effective, well tolerated and showed excellent rate of sustained virological response (SVR).

\section{INTRODUCTION}

The WHO considered $\mathrm{HCV}$ as a global health issue, with approximately $3 \%$ of the global population (roughly 170-200 million people) infected with HCV. In the US, approximately 3 million people suffers from chronic $\mathrm{HCV}$, some are still undiagnosed. The Egyptian situation is even worse. Egypt suffered the worst prevalence of hepatitis $\mathrm{C}$ in the world [1].

In Egypt, in 2015, a demographic health survey (DHS) was done in Egypt revealing $\mathrm{HCV}$ anti-body prevalence nationwide of $6.7 \%$ and HCV RNA of $4.4 \%$ in age group (159) [2].

FDA approved SOVALDI (sofosbuvir) at the end of 2013, for 
the treatment of chronic hepatitis $\mathrm{C}$ (CHC) infection as a complementary antiviral treatment regimen in subjects with $\mathrm{HCV}$ genotype 1, 2, 3 or 4 infection, for HCV Genotype 4 infection as a triple therapy with Peg INF +RBV [4].

Daclatasvir is an NS5A inhibitor with pan genotypic activity that is effective against the six major HCV genotypes with a dose of one tablet (60mg) every 24 hours. Daclatasvir with other combinations of suitable DAAs is prescribed to prevent emerging of resistance [5].

AASLD and IDSA in 2016, have recommended ledipasvir to be first line treatment in combination with Sofosbuvir for the treatment of HCV genotypes 1a, 1b, 4, 5, and 6 [6].

\section{PATIENTS AND METHODS}

This is a cross-sectional prospective study that was studied on 200 chronic hepatitis C Egyptian patients who were attending to National Hepatology \& Tropical Medicine Research Institute, Cairo Egypt, to receive the anti-HCV oral therapy in concurrence with the Egyptian guidelines of treatment in the period between April 2016 to April 2018. Informed written consent was obtained for participation in the study for every patient. The protocol of the study and the informed consent forms were approved by the human subjects committee.

Inclusion criteria: Patients $>18-<70$ years old, positive serology for $\mathrm{HCV} \mathrm{Ab}$ and $\mathrm{HCV}$ viremia, and compensated liver disease.

Exclusion criteria: Patients $<18$ or $>70$ years old, decompensated liver disease, pregnant or nursing females, coinfection with HBV, history of malignancy diagnosed or treated within 5 years, chronic use of systematically immunosuppressive agent, history of solid organ transplantation.

Patients were classified into: Group I Easy to treat: Easy to treat is defined as patient with the following: Treatment Naïve HCV patients, Serum Bilirubin < 1.2, Serum Albumin > 3.5, INR < 1.2, Platelets > 150,000. It Included 100 patients fulfilling criteria of (easy to treat) and were subdivided into two subgroups; Group Ia: 50 patients received daily Sofosbuvir $(400 \mathrm{mg})$ and Daclatasvir (60 mg) for 12 weeks. Group Ib: 50 patients received daily Sofosbuvir $(400 \mathrm{mg}$ ) and Ledipasvir (90 mg) for 12 weeks. Group II Difficult to treat: Difficult to treat is defined as patient with one or more of the following: interferon experienced, Serum Bilirubin $>1.2$, Serum Albumin < 3.5, INR >1.2, Platelets < 150,000 : It Included 100 patients fulfill criteria of (difficult to treat) and will be subdivided into two subgroups; Group IIa: 50 patients received daily Sofosbuvir (400 mg) and Daclatasvir (60 $\mathrm{mg}$ ) and daily weight-based Ribavirin (RBV) $(1000 \mathrm{mg}[<75 \mathrm{~kg}]$ to $1200 \mathrm{mg}[\geq 75 \mathrm{~kg}])$ for 12 weeks. Group IIb: 50 patients received daily Sofosbuvir (400 mg) and Ledipasvir (90 mg) and daily weight-based Ribavirin (RBV) for 12 weeks as advised by National committee for control of viral hepatitis. NCCVH.[7]

\section{Patients were subjected to the following;}

Prior to treatment: 1- Full history taking, 2Through Clinical examination 3- Abdominal ultrasonography: It was done using Toshiba SSA-340A machine with a $3.5 \mathrm{MHZ}$ curved convex probe. 4- Laboratory part of the work including:

- Biochemical tests: a- Complete blood picture: Which were done on Sysmex (Germany). b- Liver and kidney function tests: Which were done on Cobas c311 auto analyzer (Germany) using Roch reagent kits, prothrombin time, concentration and INR, fasting blood sugar, HBA1c for diabetics, Alfa feto protein, HCV PCR Quantitative, HBsAg.

-Fib 4 for assessment of fibrosis: FIB-4 score was used as a non-invasive routine biochemical method to assess fibrosis stage. It was calculated for patients before and after the end treatment.

\begin{tabular}{|c|c|}
\hline FIB-4 & $\begin{array}{l}\text { Age (years) } \times \text { AST }[\mathrm{U} / \mathrm{l}] / \text { (platelets } \\
\left.\left[10^{9} / \mathrm{L}\right] \times(\mathrm{ALT}[\mathrm{U} / \mathrm{L}])^{1 / 2}\right)\end{array}$ \\
\hline
\end{tabular}

-Monitoring of treatment safety: For all patients: liver biochemical profile, complete blood count and creatinine were tested every visit (1 month). Patients were asked about the commonly reported adverse events as headache, nausea, insomnia, pruritus, fatigue, rash, photosensitivity.

-Monitoring of treatment efficacy at the end: All patients were followed up by $\mathrm{CBC}$, liver functions, kidney functions at weeks 4,8 , end of treatment and week 24. Quantitative PCR for HCV was done at weeks $0,12,24$ (SVR 12, 24) Virologic response was considered when $\mathrm{HCV}$ RNA is less than the lower limit of detection at end of treatment and post treatment at week 24 
-Statistical Analysis: Analysis of data was performed using SPSS v. 16. (Statistical Package for Scientific Studies) for Windows.

\section{RESULTS}

Table (1): Comparison between laboratory data pretreatment and post treatment in Group Ia group.

\begin{tabular}{|c|c|c|c|c|c|c|}
\hline & \multicolumn{2}{|c|}{ Group Ia } & \multirow{2}{*}{$\begin{array}{c}\text { Mean difference } \\
\pm \mathrm{SE}\end{array}$} & \multirow{2}{*}{ Test value } & \multirow{2}{*}{ P-value } \\
\hline & & Pre & Post & & & \\
\hline \multirow[b]{2}{*}{ ALT unit/dl } & Mean \pm SD & $59.23 \pm 37.45$ & $42.56 \pm 20.84$ & \multirow{2}{*}{$-16.67 \pm 5.13$} & \multirow{2}{*}{3.251} & \multirow[b]{2}{*}{0.002} \\
\hline & Range & $12.38-236.67$ & $21-95.26$ & & & \\
\hline \multirow{2}{*}{ AST unit/dl } & Mean \pm SD & $66.62 \pm 54.41$ & $49.95 \pm 22.43$ & \multirow{2}{*}{$-16.66 \pm 7.59$} & \multirow{2}{*}{3.701} & \multirow{2}{*}{0.001} \\
\hline & Range & $17.3-346.32$ & $20-90$ & & & \\
\hline \multirow{2}{*}{ Albumin gm/dl } & Mean \pm SD & $4.14 \pm 0.36$ & $3.91 \pm 0.31$ & \multirow{2}{*}{$-0.23 \pm 0.06$} & \multirow{2}{*}{2.194} & \multirow{2}{*}{0.033} \\
\hline & Range & $3.5-4.9$ & $3.5-4.3$ & & & \\
\hline \multirow{2}{*}{ Total BILIRUBIN mg/dI } & Mean \pm SD & $0.92 \pm 0.32$ & $0.73+0.21$ & \multirow{2}{*}{$0.18 \pm 0.05$} & \multirow{2}{*}{3.524} & \multirow{2}{*}{0.001} \\
\hline & Range & $0.27-1.41$ & $0.3+1.1$ & & & \\
\hline \multirow{2}{*}{ PC \% } & Mean \pm SD & $90.72 \pm 8.19$ & $93.76 \pm 7.38$ & \multirow{2}{*}{$3.04 \pm 1.49$} & \multirow{2}{*}{-2.038} & \multirow{2}{*}{0.047} \\
\hline & Range & $78-100$ & $82-100$ & & & \\
\hline \multirow{2}{*}{ INR } & Mean \pm SD & $1.03 \pm 0.05$ & $1.10 \pm 0.18$ & \multirow{2}{*}{$0.1 \pm 0.026$} & \multirow{2}{*}{2.910} & \multirow[b]{2}{*}{0.05} \\
\hline & Range & $1-1.1$ & $1-1.2$ & & & \\
\hline \multirow{2}{*}{ AFP IU/L } & Mean \pm SD & $10.82 \pm 10.78$ & $10.28 \pm 1.71$ & \multirow{2}{*}{$0.02 \pm 1.34$} & \multirow{2}{*}{-0.016} & \multirow{2}{*}{0.987} \\
\hline & Range & $3-57$ & $8-20$ & & & \\
\hline
\end{tabular}

This table shows that, there was a statistically significant improvement in all liver functions studied, post treatment in comparison to pretreatment in GIa, while AFP show insignificant changes.

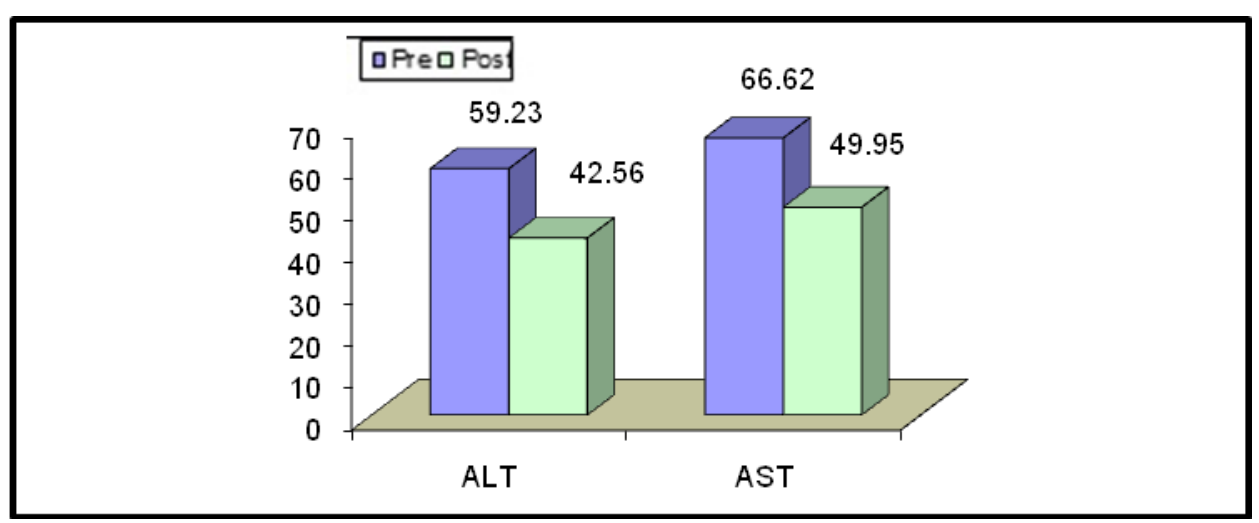

Fig. (1): Shows ALT, AST in Group Ia pre and post treatment

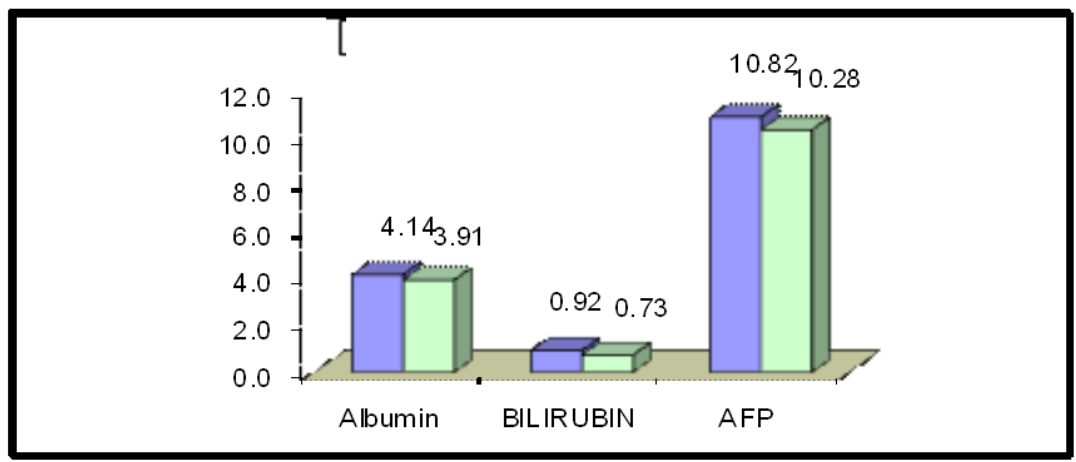

Fig. (2): shows Albumin, bilirubin, AFP in Group Ia pre and post treatment. 


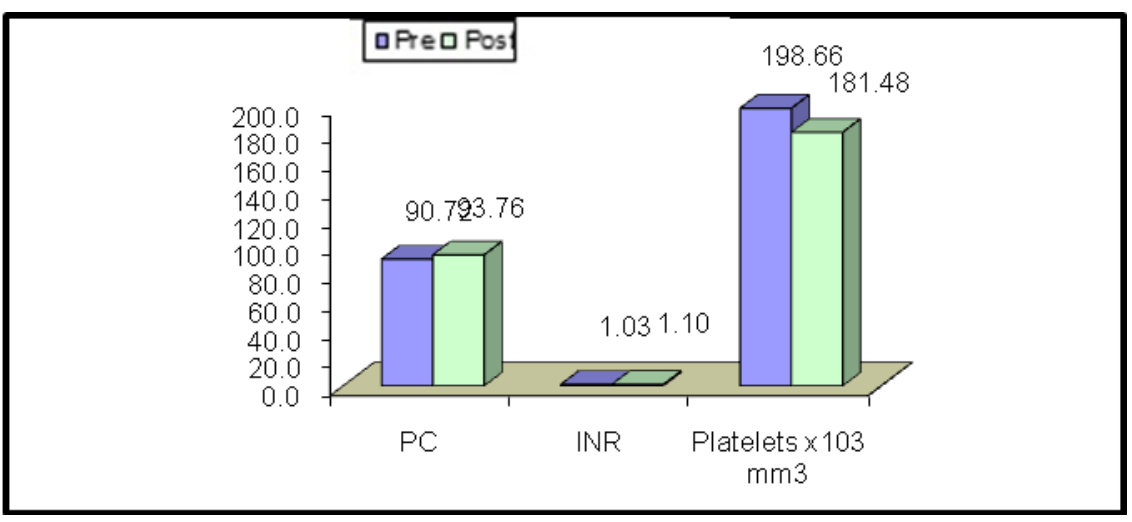

Fig. (3): Shows platelets, Prothrombin concentration, INR in GroupIa pre and post treatment.

Table (2): Comparison between the Complete blood Count data pretreatment and post treatment in Group Ia group

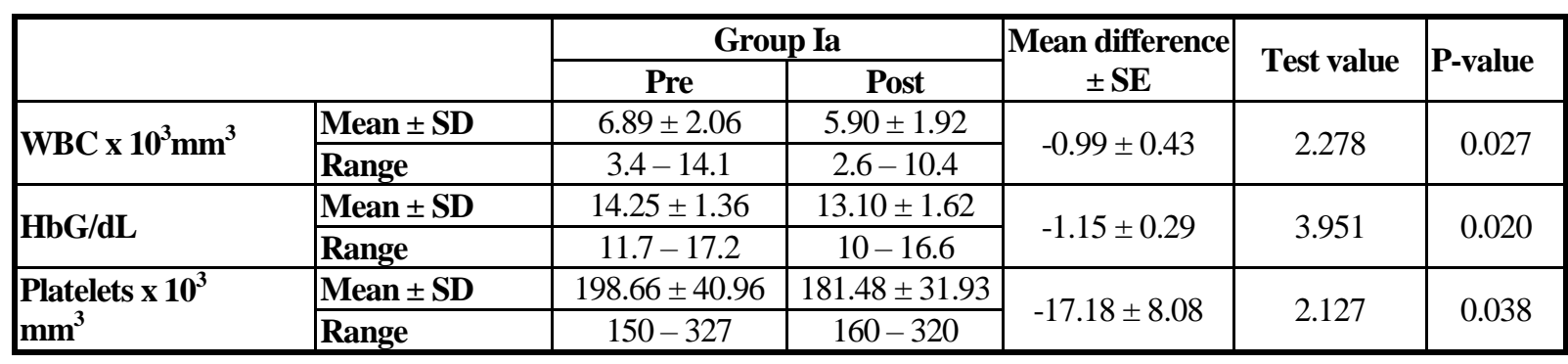

This table shows CBC results there was significant difference between pretreatment and post treatment in GIa

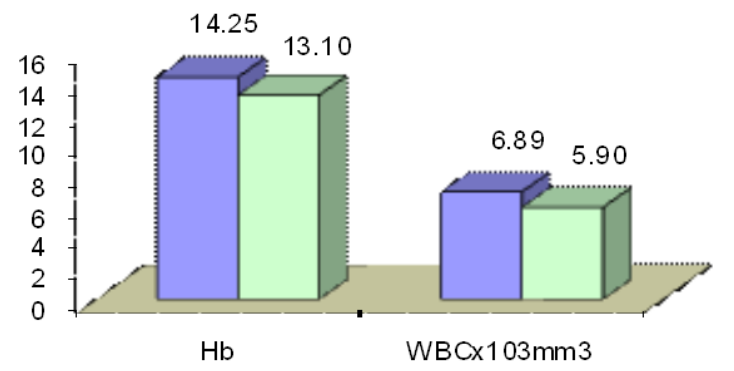

Fig. (4): Shows hemoglobin and white blood cells in Group Ia pre and post treatment.

Table (3): Comparison between laboratory data pretreatment and post treatment in Group I b group

\begin{tabular}{|c|c|c|c|c|c|c|}
\hline & GIb & Pre & Post & $\begin{array}{c}\text { Mean difference } \\
\pm \mathrm{SE}\end{array}$ & t-test & p-value \\
\hline \multirow{2}{*}{ ALT } & Mean \pm SD & $59.40 \pm 33.40$ & $46.28 \pm 25.17$ & \multirow{2}{*}{$-13.12 \pm 6.13$} & \multirow{2}{*}{2.139} & \multirow{2}{*}{0.037} \\
\hline & Range & $10-154.44$ & $18-106.86$ & & & \\
\hline \multirow{2}{*}{ AST } & Mean \pm SD & $55.16 \pm 26.76$ & $54.23 \pm 19.29$ & \multirow{2}{*}{$-0.94 \pm 4.46$} & \multirow{2}{*}{0.210} & \multirow{2}{*}{0.834} \\
\hline & Range & $7.57-140$ & $30-89$ & & & \\
\hline \multirow{2}{*}{ Albumin g/dL } & Mean \pm SD & $3.79 \pm 0.23$ & $4.13+0.40$ & \multirow{2}{*}{$-0.34 \pm 0.06$} & \multirow{2}{*}{5.312} & \multirow{2}{*}{0.000} \\
\hline & Range & $3.5-4.3$ & $3.5-4.9$ & & & \\
\hline \multirow{2}{*}{$\begin{array}{l}\text { Total BILIRUBIN } \\
\text { mg/dL }\end{array}$} & Mean \pm SD & $0.91 \pm 0.30$ & $0.72 \pm 0.26$ & \multirow{2}{*}{$0.18 \pm 0.06$} & \multirow{2}{*}{-3.146} & \multirow{2}{*}{0.003} \\
\hline & Range & $0.27-1.2$ & $0.3-1.1$ & & & \\
\hline \multirow{2}{*}{$\mathbf{P C}$} & Mean \pm SD & $91.00 \pm 7.46$ & $91.36 \pm 4.04$ & \multirow{2}{*}{$0.36 \pm 1.27$} & \multirow{2}{*}{$\mid-0.283$} & \multirow{2}{*}{0.778} \\
\hline & Range & $82-100$ & $88-100$ & & & \\
\hline \multirow{2}{*}{ INR } & Mean \pm SD & $1.03 \pm 0.05$ & $1.18 \pm 0.19$ & \multirow{2}{*}{$0.15 \pm 0.03$} & \multirow{2}{*}{-5.268} & \multirow{2}{*}{0.000} \\
\hline & Range & $1-1.1$ & $1-1.2$ & & & \\
\hline \multirow{2}{*}{ AFP } & Mean \pm SD & $10.26 \pm 13.12$ & $10.53 \pm 3.13$ & \multirow{2}{*}{$0.02 \pm 2.14$} & \multirow{2}{*}{0.010} & \multirow{2}{*}{0.992} \\
\hline & Range & $5-77$ & $6-30$ & & & \\
\hline
\end{tabular}

Nouh et al., Afro-Egypt J Infect Endem Dis 2020;10(2):163-173

https://aeji.journals.ekb.eg/

http://mis.zu.edu.eg/ajied/home.aspx 
This is table shows there was significant decrease in, bilirubin, ALT, while there was significant increase in s. Albumin and INR post treatment in comparison to pretreatment in GIb.

Table (4): Comparison between Complete Blood Count pretreatment and post treatment in Group Ib

\begin{tabular}{|c|c|c|c|c|c|c|}
\hline GIb & Group 1 & Pre & Post & $\begin{array}{c}\text { Mean difference } \\
\pm \mathrm{SE}\end{array}$ & t-test & p-value \\
\hline \multirow{2}{*}{$\mathrm{WBC} \times 10^{3} / \mathrm{mm}^{3}$} & Mean \pm SD & $6.32 \pm 1.79$ & $5.06 \pm 1.86$ & \multirow{2}{*}{$-1.26 \pm 0.38$} & \multirow{2}{*}{3.282} & \multirow{2}{*}{0.002} \\
\hline & Range & $3.6-11.4$ & $2.5-10.4$ & & & \\
\hline \multirow{2}{*}{ Hb/GL } & Mean \pm SD & $13.97 \pm 1.54$ & $13.33 \pm 1.44$ & \multirow{2}{*}{$-0.64 \pm 0.30$} & \multirow{2}{*}{2.100} & \multirow{2}{*}{0.041} \\
\hline & Range & $10-16.6$ & $10.4-16$ & & & \\
\hline \multirow{2}{*}{ Platelets $x 10^{3} / \mathrm{mm}^{3}$} & Mean \pm SD & $196.76 \pm 44.47$ & $183.26 \pm 34.93$ & \multirow{2}{*}{$-13.5 \pm 7.99$} & \multirow{2}{*}{1.689} & \multirow{2}{*}{0.097} \\
\hline & Range & $165-341$ & $165-338$ & & & \\
\hline
\end{tabular}

This table shows $\mathrm{CBC}$ results as there was no significant difference in the platelet count between pretreatment and post treatment in GIb except WBCs there was a statistically significant decrease.

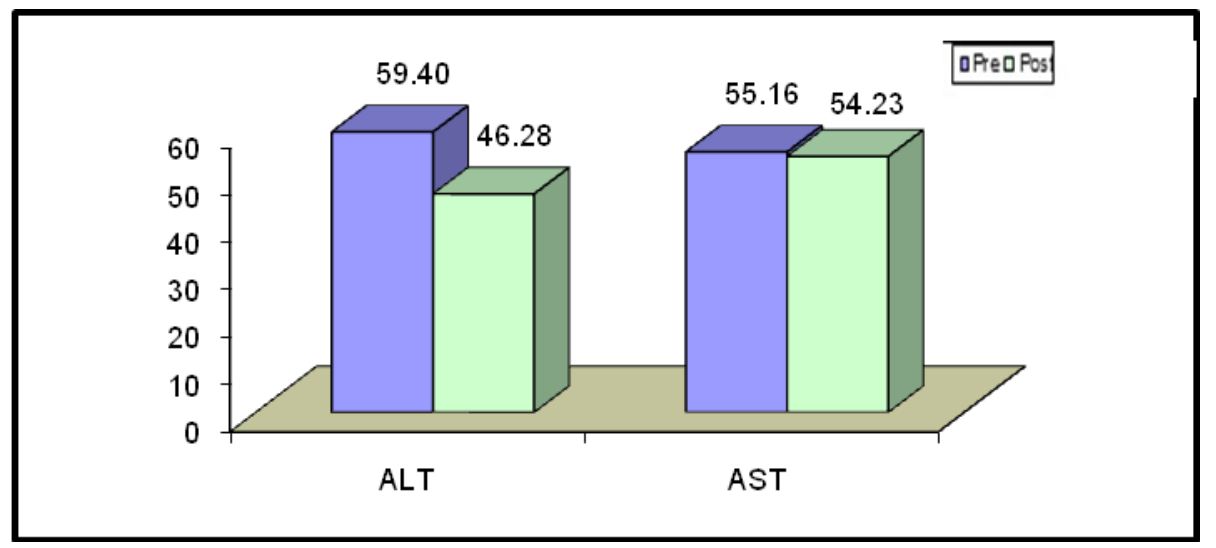

Fig. (5): Comparison between ALT, AST pretreatment and post treatment in Group Ib

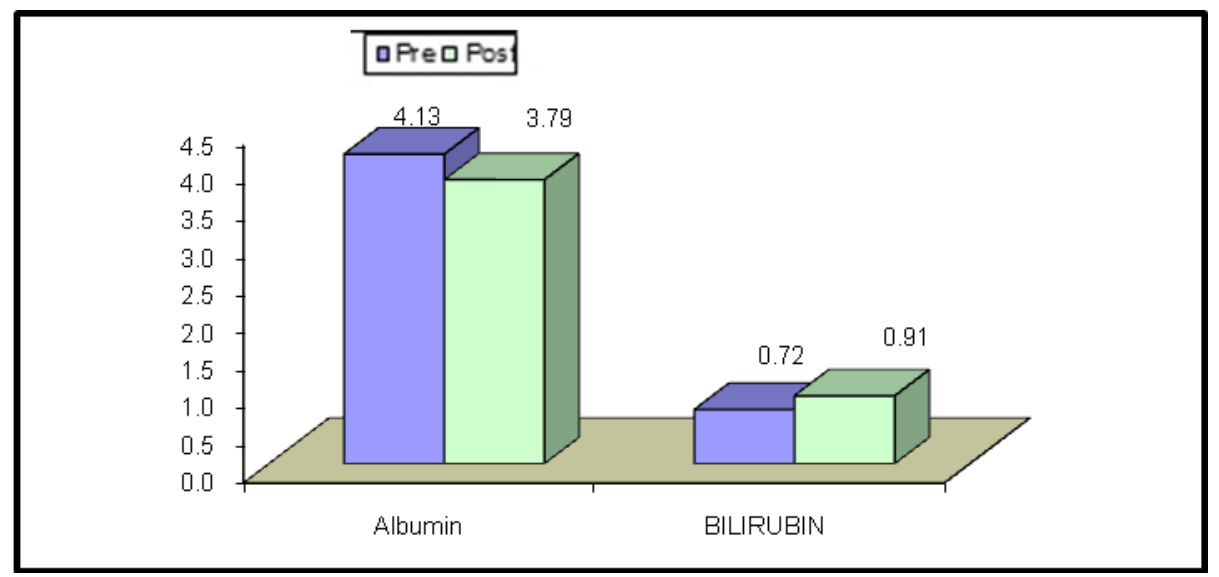

Fig.(6): Comparison between Albumin and bilirubin pretreatment and post treatment in Group Ib 


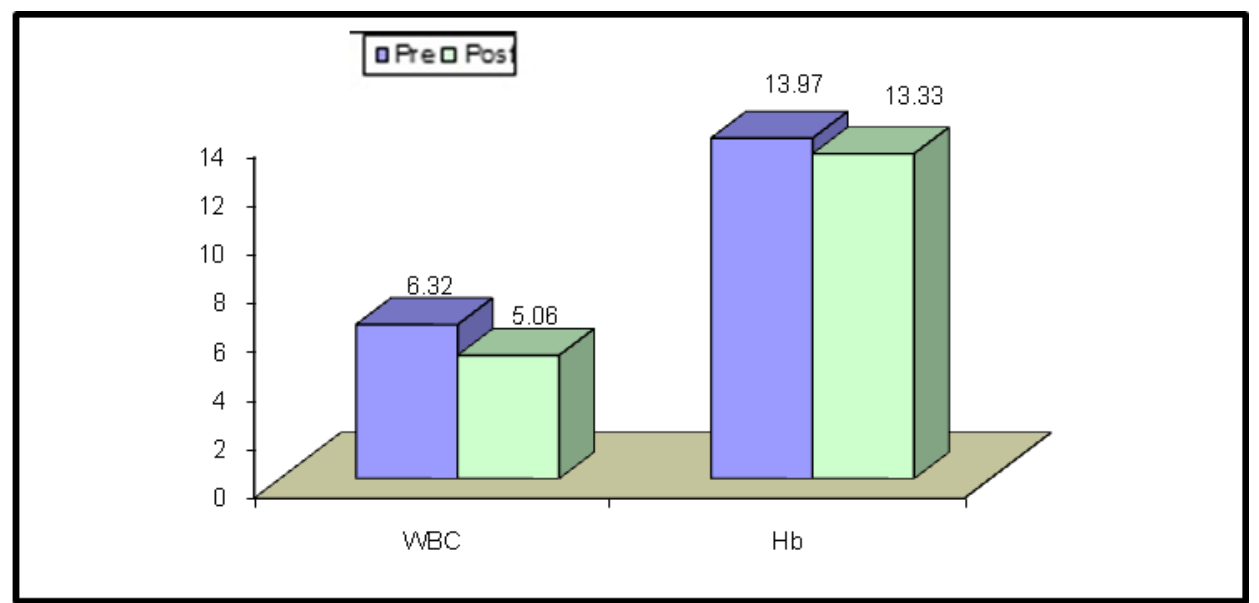

Fig. (7): Comparison between white blood cells and hemoglobin pretreatment and post treatment in Group Ib

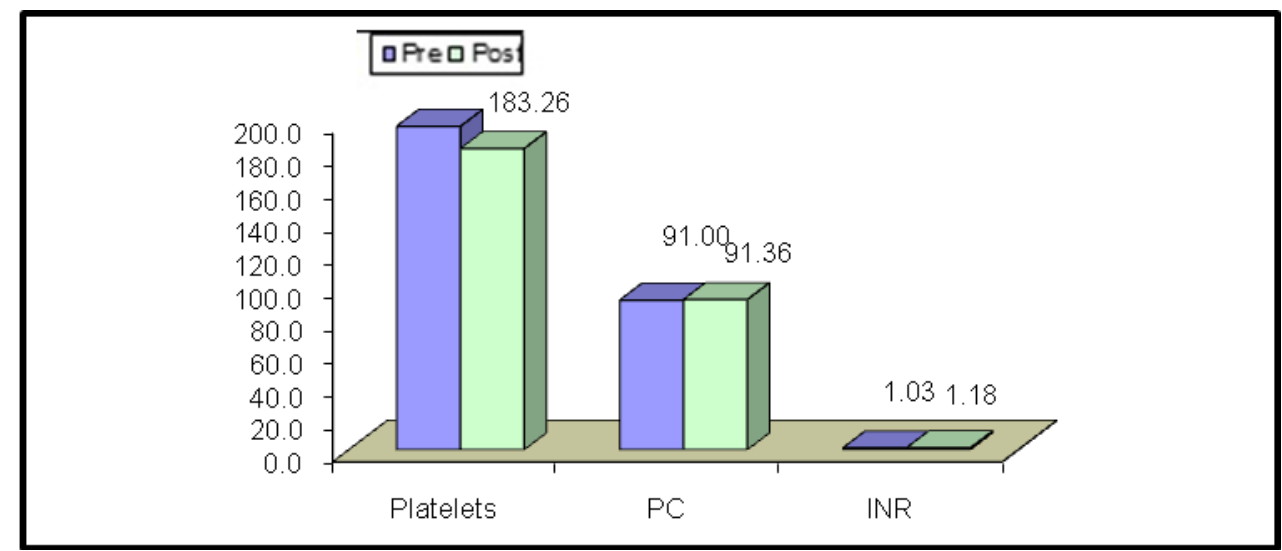

Fig. (8): Comparison between platelets, Prothrombin concentration, INR pretreatment and post treatment in Group Ib

Table (5): Comparison between laboratory data pretreatment and post treatment in Group IIa

\begin{tabular}{|c|c|c|c|c|c|c|}
\hline & & \multicolumn{2}{|c|}{ Group IIa } & \multirow{2}{*}{ Mean diff \pm SE } & \multirow{2}{*}{ Test value } & \multirow{2}{*}{ P-value } \\
\hline & & Pre & Post & & & \\
\hline \multirow{2}{*}{ ALT } & Mean \pm SD & $69.46 \pm 47.28$ & $43.07 \pm 18.51$ & \multirow{2}{*}{$-26.39 \pm 7.35$} & \multirow{2}{*}{3.591} & \multirow{2}{*}{0.001} \\
\hline & Range & $10-270$ & $9-87$ & & & \\
\hline \multirow{2}{*}{ AST } & Mean \pm SD & $87.89 \pm 54.18$ & $54.94 \pm 20.47$ & \multirow{2}{*}{$-32.95 \pm 7.74$} & \multirow{2}{*}{4.259} & \multirow{2}{*}{0.000} \\
\hline & Range & $10-282$ & $22-93$ & & & \\
\hline \multirow{2}{*}{ Albumin g/dL } & Mean \pm SD & $3.52 \pm 0.57$ & $3.10 \pm 0.29$ & \multirow{2}{*}{$-0.42 \pm 0.08$} & \multirow{2}{*}{5.144} & \multirow{2}{*}{0.000} \\
\hline & Range & $2.8-4.9$ & $2.8-4$ & & & \\
\hline \multirow{2}{*}{ Total BILIRUBIN mg/dL } & Mean \pm SD & $1.21 \pm 0.60$ & $1.18 \pm 0.55$ & \multirow{2}{*}{$-0.03 \pm 0.09$} & \multirow{2}{*}{0.343} & \multirow{2}{*}{0.733} \\
\hline & Range & $0.3-2.5$ & $0.5-4$ & & & \\
\hline \multirow{2}{*}{ PC } & Mean \pm SD & $76.74 \pm 12.26$ & $90.34 \pm 11.20$ & \multirow{2}{*}{$13.60 \pm 2.44$} & \multirow{2}{*}{-5.572} & \multirow{2}{*}{0.000} \\
\hline & Range & $62-100$ & $65-100$ & & & \\
\hline \multirow{2}{*}{ INR } & Mean \pm SD & $1.25 \pm 0.18$ & $1.05 \pm 0.07$ & \multirow{2}{*}{$-0.21 \pm 0.03$} & \multirow{2}{*}{7.271} & \multirow{2}{*}{0.000} \\
\hline & Range & $1-1.6$ & $1-1.32$ & & & \\
\hline \multirow{2}{*}{ AFP IUL } & Mean \pm SD & $18.74 \pm 18.42$ & $12.02 \pm 9.89$ & \multirow{2}{*}{$-6.72 \pm 3.03$} & \multirow{2}{*}{2.221} & \multirow{2}{*}{0.031} \\
\hline & Range & $3-82$ & $10-60$ & & & \\
\hline
\end{tabular}

This is table shows there was significant decrease in AST, ALT, INR and AFP, while there was significant increase in PC post treatment in comparison to pretreatment in GIIa \& insignificant changes in bilirubin. 
Table (6): Comparison between Complete Blood Count pretreatment and post treatment in Group IIa

\begin{tabular}{|c|c|c|c|c|c|c|}
\hline & \multicolumn{2}{|c|}{ Group IIa } & \multirow{2}{*}{ Mean diff $\pm \mathrm{SE}$} & \multirow{2}{*}{ Test value } & \multirow{2}{*}{ P-value } \\
\hline & & Pre & Post & & & \\
\hline \multirow{2}{*}{$\mathrm{WBC} \times 10^{3} \mathrm{~mm}^{3}$} & Mean \pm SD & $5.04 \pm 2.02$ & $6.53 \pm 8.83$ & \multirow{2}{*}{$1.49 \pm 1.30$} & \multirow{2}{*}{-1.143} & \multirow{2}{*}{0.259} \\
\hline & Range & $2.6-14.1$ & $3.4-67$ & & & \\
\hline \multirow{2}{*}{ Hb G/L } & Mean \pm SD & $13.27 \pm 1.72$ & $13.53 \pm 1.40$ & \multirow{2}{*}{$0.26 \pm 0.27$} & \multirow{2}{*}{-0.960} & \multirow{2}{*}{0.342} \\
\hline & Range & $10.4-16.6$ & $10-15$ & & & \\
\hline \multirow{2}{*}{ Platelets $\times 10^{3} \mathrm{~mm}^{3}$} & Mean \pm SD & $95.46 \pm 32.80$ & $161.64 \pm 29.98$ & \multirow{2}{*}{$66.18 \pm 6.51$} & \multirow{2}{*}{-10.172} & \multirow{2}{*}{0.000} \\
\hline & Range & $65-248$ & $70-230$ & & & \\
\hline
\end{tabular}

This table shows there was highly significant increase in platelet count, post treatment in comparison to pre treatment, while there was no significant differences in WBCs and hemoglobin in GIIa

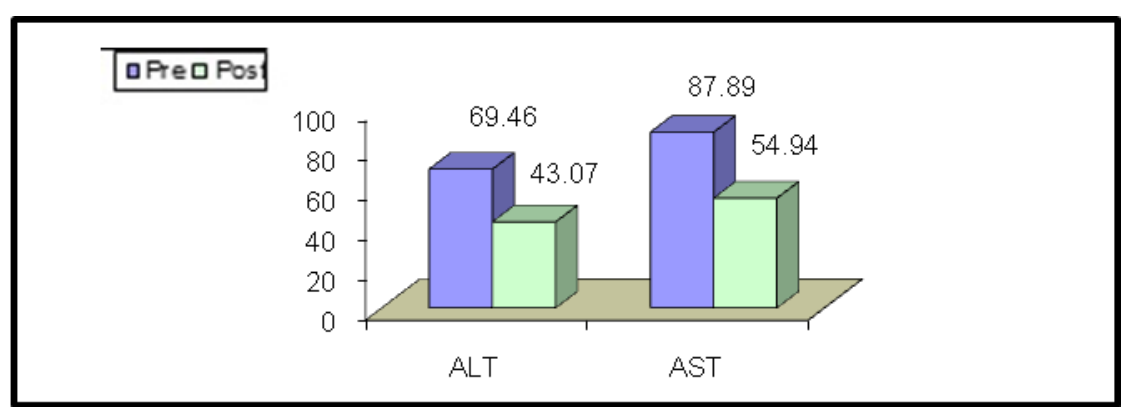

Fig. (9): Comparison between ALT, AST pretreatment and post treatment in Group IIa

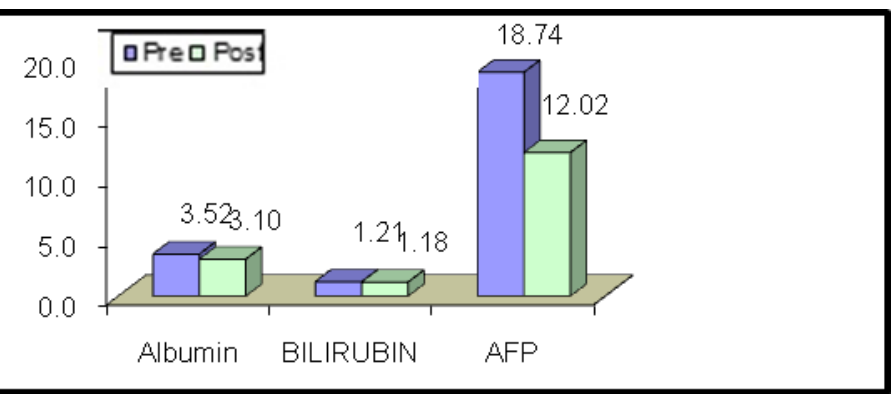

Fig. (90): Comparison between Albumin, bilirubin, AFP pretreatment and post treatment in Group IIa

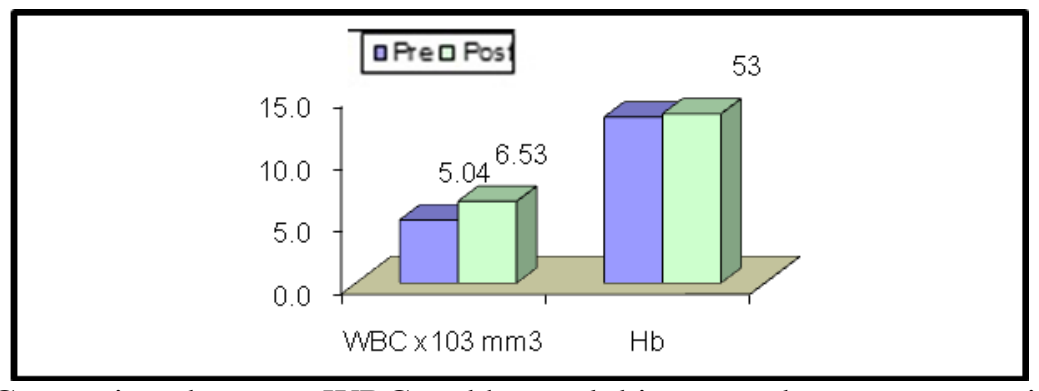

Fig. (101): Comparison between WBC and hemoglobin pre and post treatment in Group IIa. 


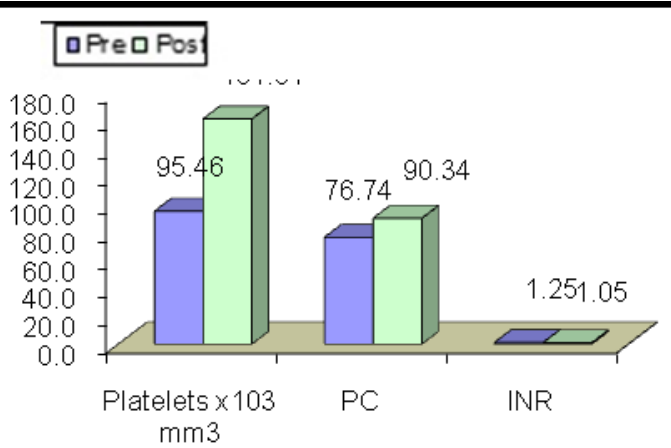

Fig. (112): Comparison between platelets, PC, INR pre and post treatment in Group IIa

Table (7): Comparison between laboratory data pretreatment and post treatment in Group IIb

\begin{tabular}{|c|c|c|c|c|c|c|}
\hline & \multicolumn{2}{|c|}{ Group IIb } & \multirow{2}{*}{ Mean diff $\pm \mathrm{SE}$} & \multirow{2}{*}{ Test value } & \multirow{2}{*}{ P-value } \\
\hline & & Pre & Post & & & \\
\hline \multirow[b]{2}{*}{ ALT } & Mean \pm SD & $55.17 \pm 33.05$ & $43.37 \pm 20.64$ & \multirow{2}{*}{$-11.80 \pm 4.99$} & \multirow{2}{*}{2.362} & \multirow{2}{*}{0.022} \\
\hline & \begin{tabular}{|l|} 
Range \\
\end{tabular} & $13.6-160$ & $7-98$ & & & \\
\hline \multirow{2}{*}{ AST } & Mean \pm SD & $71.40 \pm 35.82$ & $45.45 \pm 21.76$ & \multirow{2}{*}{$-25.95 \pm 5.83$} & \multirow{2}{*}{4.454} & \multirow{2}{*}{0.000} \\
\hline & Range & $5-169$ & $15-75$ & & & \\
\hline \multirow{2}{*}{ Albumin g/dL } & Mean \pm SD & $3.66 \pm 0.60$ & $3.09 \pm 0.50$ & \multirow{2}{*}{$-0.57 \pm 0.12$} & \multirow{2}{*}{4.822} & \multirow{2}{*}{0.000} \\
\hline & Range & $2.8-4.9$ & $1-4.6$ & & & \\
\hline \multirow{2}{*}{$\begin{array}{l}\text { Total BILIRUBIN } \\
\mathrm{mg} / \mathrm{dL}\end{array}$} & Mean \pm SD & $1.20 \pm 0.52$ & $1.06 \pm 0.18$ & \multirow{2}{*}{$-0.14 \pm 0.08$} & \multirow{2}{*}{1.789} & \multirow{2}{*}{0.080} \\
\hline & Range & $0.4-2.4$ & $0.7-1.6$ & & & \\
\hline \multirow[b]{2}{*}{ PC } & Mean \pm SD & $80.90 \pm 13.32$ & $90.82 \pm 11.02$ & \multirow{2}{*}{$9.92 \pm 2.32$} & \multirow{2}{*}{-4.280} & \multirow{2}{*}{0.000} \\
\hline & \begin{tabular}{|l} 
Range \\
\end{tabular} & $60-100$ & $69-100$ & & & \\
\hline \multirow{2}{*}{ INR } & Mean \pm SD & $1.21 \pm 0.20$ & $1.04 \pm 0.08$ & \multirow{2}{*}{$-0.17 \pm 0.03$} & \multirow{2}{*}{5.628} & \multirow{2}{*}{0.000} \\
\hline & Range & $1-1.7$ & $1-1.36$ & & & \\
\hline \multirow{2}{*}{ AFP IUL } & Mean \pm SD & $15.78 \pm 14.86$ & $13.90 \pm 12.16$ & \multirow{2}{*}{$-1.88 \pm 2.38$} & \multirow{2}{*}{0.789} & \multirow{2}{*}{0.434} \\
\hline & Range & $7-75$ & $9-50$ & & & \\
\hline
\end{tabular}

This table shows there was significant decrease in ALT, AST, s. bilirubin, s. albumin and INR, while there was significant increase in PC post treatment in comparison to pretreatment in GIIb.

Table (8): Comparison between the CBC pre and post treatment in Group IIb

\begin{tabular}{|c|c|c|c|c|c|c|}
\hline & \multicolumn{2}{|c|}{ Group IIb } & \multirow{2}{*}{ Mean diff $\pm \mathrm{SE}$} & \multirow{2}{*}{ Test value } & \multirow{2}{*}{ P-value } \\
\hline & & Pre & Post & & & \\
\hline \multirow{2}{*}{$\mathrm{WBC} \times 10^{3} \mathrm{~mm}^{3}$} & Mean \pm SD & $4.82 \pm 1.91$ & $5.31 \pm 1.21$ & \multirow{2}{*}{$0.49 \pm 0.30$} & \multirow[b]{2}{*}{-1.614} & \multirow{2}{*}{0.113} \\
\hline & \begin{tabular}{|l|} 
Range \\
\end{tabular} & $2.4-11.2$ & $3.4-8$ & & & \\
\hline \multirow{2}{*}{ Hb/GL } & Mean \pm SD & $13.41 \pm 1.28$ & $13.64 \pm 1.44$ & \multirow{2}{*}{$0.23 \pm 0.27$} & \multirow{2}{*}{-0.848} & \multirow{2}{*}{0.401} \\
\hline & Range & $10.6-16.8$ & $10-15$ & & & \\
\hline \multirow{2}{*}{ Platelets $x 10^{3} \mathrm{~mm}^{3}$} & Mean \pm SD & $112.48 \pm 49.12$ & $164.34 \pm 25.60$ & \multirow{2}{*}{$51.86 \pm 7.59$} & \multirow{2}{*}{-6.829} & \multirow{2}{*}{0.000} \\
\hline & Range & $74-260$ & $80-212$ & & & \\
\hline
\end{tabular}

This table shows there was significant increase in platelet count post treatment in comparison to pretreatment in GIIb 


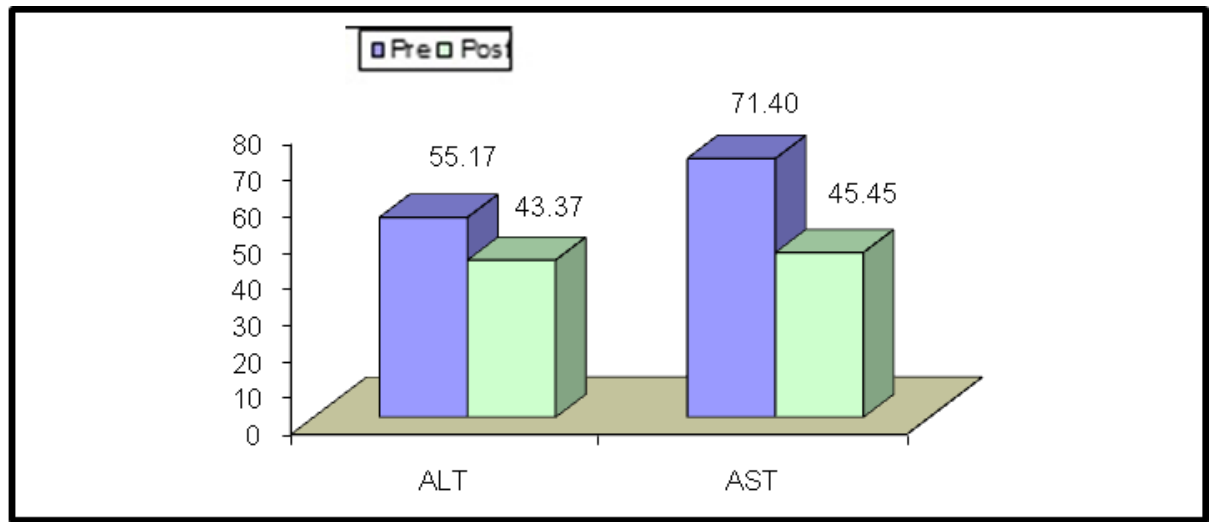

Fig. (13): Comparison between ALT, AST pre and post treatment in Group II b

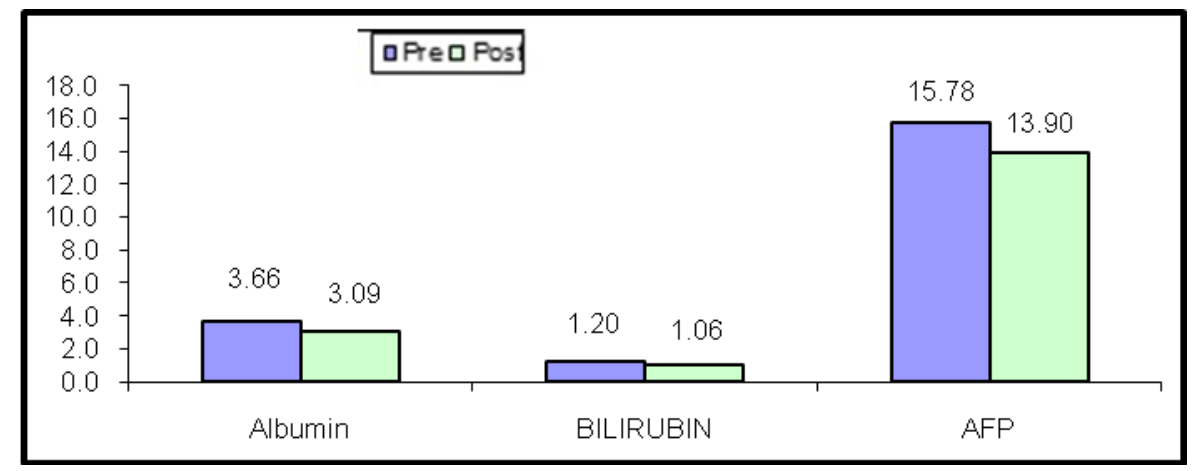

Fig. (124): Comparison between Albumin, bilirubin, AFP pre and post treatment in Group II b

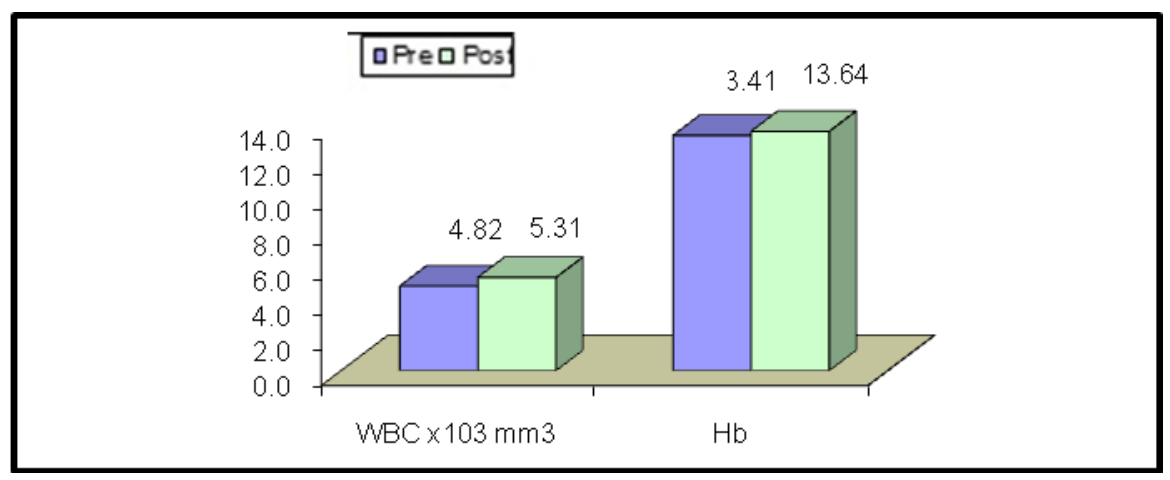

Fig. (135): Comparison between WBC and hemoglobin pre and post treatment in Group II

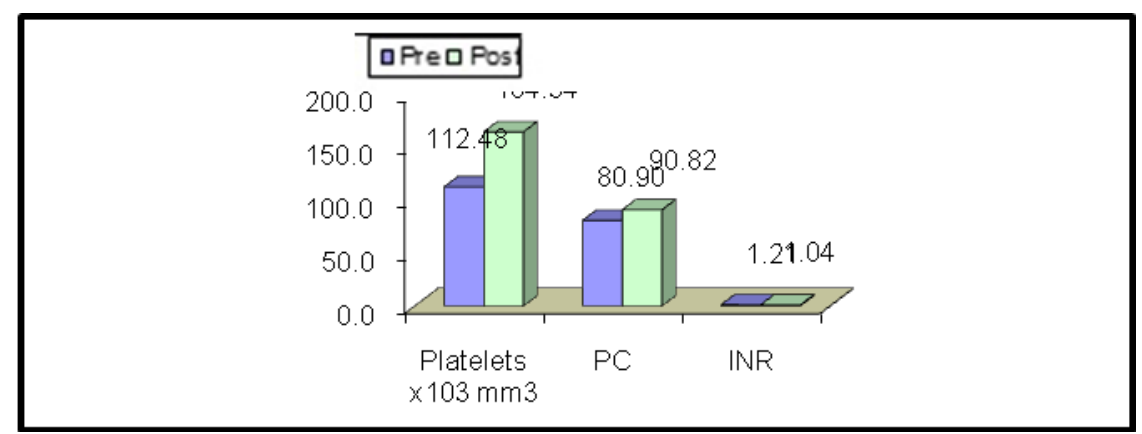

Fig. (146): Comparison between platelets, PC, INR pre and post treatment in Group II b 
Table (9): FIB4 calculation in Group I pre and post treatment

\begin{tabular}{|c|c|c|c|c|c|c|}
\hline \multirow{2}{*}{ Fib4 Calculation } & \multicolumn{2}{|c|}{ Group Ia } & \multirow{2}{*}{ P-value } & \multicolumn{2}{|c|}{ Group Ib } & \multirow{2}{*}{ P-value } \\
\hline & Baseline & $24 w$ & & Baseline & $24 w$ & \\
\hline Mean \pm SD & $2.23 \pm 1.12$ & $0.67 \pm 0.31$ & \multirow{2}{*}{$<0.001$} & $2.13 \pm 1.11$ & $1.12 \pm 0.59$ & \multirow{2}{*}{$<0.001$} \\
\hline Range & $0.33-5.12$ & $0.14-1.77$ & & $0.38-4.73$ & $0.2-2.64$ & \\
\hline
\end{tabular}

There was highly significant decrease in FIB4 calculation at week 24 in comparison to the baseline in GIa \& GIb.

Table (50): FIB4 calculation in Group II pre and post treatment

\begin{tabular}{|c|c|c|c|c|c|c|}
\hline \multirow{2}{*}{ Fib4 Calculation } & \multicolumn{2}{|c|}{ Group IIa } & \multirow{2}{*}{ P-value } & \multicolumn{2}{|c|}{ Group IIb } & \multirow{2}{*}{ P-value } \\
\hline & Baseline & 24 w & & Baseline & $24 w$ & \\
\hline Mean \pm SD & $6.83 \pm 4.55$ & $4.81 \pm 3.30$ & \multirow{2}{*}{0.063} & $6.12 \pm 4.02$ & $5.83 \pm 3.45$ & \multirow{2}{*}{0.699} \\
\hline range & $0.58-25.51$ & $0.38-21.97$ & & $0.22-19.26$ & $0.17-18.43$ & \\
\hline
\end{tabular}

There was no statistically significant decrease in FIB4 calculation at week 24 in comparison to the baseline in GIIa \& GIIb

Table (61): Shows comparison between the PCR results in the studied groups at week 12 and 24 .

\begin{tabular}{|c|c|c|c|c|c|c|c|c|c|c|c|}
\hline & \multicolumn{4}{|c|}{ Group I } & \multirow{3}{*}{ P-value* } & \multicolumn{4}{|c|}{ Group II } & \multirow{3}{*}{ P-value* } \\
\hline & & \multicolumn{2}{|c|}{ GIa } & \multicolumn{2}{|c|}{ GIb } & & \multicolumn{2}{|c|}{ GIIa } & \multicolumn{2}{|c|}{ GIIb } & \\
\hline & & No. & $\%$ & No. & $\%$ & & No. & $\%$ & No. & $\%$ & \\
\hline \multirow{2}{*}{ HCVRNAW12 } & Negative & 50 & $100 \%$ & 49 & $98 \%$ & \multirow{2}{*}{0.315} & 50 & $100 \%$ & 49 & $98.0 \%$ & \multirow{2}{*}{0.315} \\
\hline & Positive & $\overline{0}$ & $0.0 \%$ & 1 & $2.0 \%$ & & 0 & $0.0 \%$ & 1 & $2.0 \%$ & \\
\hline \multirow{2}{*}{ - HCVRNAW24 } & Negative & 50 & $100.0 \%$ & 49 & $98.0 \%$ & \multirow{2}{*}{0.315} & 49 & $98.0 \%$ & 48 & $96.0 \%$ & \multirow{2}{*}{0.558} \\
\hline & Positive & 0 & $0.0 \%$ & 1 & $2.0 \%$ & & 1 & $2.0 \%$ & 2 & $4.0 \%$ & \\
\hline
\end{tabular}

This table shows the results of quantitative PCR at wk 12 and wk 24

- In GIa : showed $100 \%$ cure (-ve PCR) at wk12 and wk 24, while in GIb : showed $98 \%$ cure (-ve PCR) at wk12 and wk 24. In GIIa : showed $100 \%$ cure (-ve PCR) at wk12 and 98\% at wk 24, while in GIIb : showed 98\% cure (-ve PCR) at wk12 and 96 at wk 24. There was no statistical difference in the cure rate in different groups of the study.

\section{DISCUSSION}

As regard liver functions pre and post treatment, there was significant decrease in ALT, AST and s. bilirubin post treatment in comparison to pretreatment in GIa, while there was significant decrease in ALT and s. albumin and significant increase in $\mathrm{s}$. bilirubin and INR post-treatment in comparison to pretreatment in GIb.

Also there was significant decrease in ALT, AST and INR, while there was significant increase in PC post-treatment in comparison to pretreatment in GIIa, while there was significant decrease in ALT, AST, s. bilirubin, s. albumin and INR, and there was significant increase in PC post-treatment in comparison to pretreatment in GIIb. So we concluded that there was no significant deference between two regimens.
As regard comparison of $\mathrm{CBC}$ results post treatment and pretreatment there was no significant difference between pre-treatment and post-treatment in GIa and GIb, while there was highly significant increase in platelet count, post treatment in comparison to pre-treatment in GIIa and GIIb indicate that there was no significant deference between two regimens.

As regard FIB4 calculation, there was highly significant decrease in FIB4 at week 24 in comparison to the baseline in GIa (2.23 to 0.67) and GIb (2.1 to 1.12) (easy to treat), while there was no statistically significant deference in FIB4 calculation at week 24 in GIIa and GIIb (difficult to treat). This can be explained by the fact that in advanced liver disease there is little improvement of liver condition after DAAs therapy in 24 wks period.

This was in agreement with Wong et al.[8] whose study showed Among 2691 patients, all

Nouh et al., Afro-Egypt J Infect Endem Dis 2020;10(2):163-173

https://aeji.journals.ekb.eg/

http://mis.zu.edu.eg/ajied/home.aspx 
markers of disease severity demonstrated sustained improvements from pre-treatment to 4 years post-treatment.

As regard the virological response by quantitative PCR in the studied groups In GIa: showed $100 \%$ cure (-ve PCR) at wk12 and wk 24, while in GIb: showed 98\% cure (-ve PCR) at wk12 and wk 24.

Also In GIIa: showed $100 \%$ cure (-ve PCR) at wk12 and $98 \%$ at wk 24, while in GIlb showed $98 \%$ cure (-ve PCR) at wk12 and 96 at wk 24.

This goes in agreement with Shiha et al. [9] who found that SVR is $96 \%$ after treatment of 177 patients with SOF+DAC for 3 months in comparison to our study SVR is $100 \%$.

Also, this was in agreement with Ahmed et al. [10] who found that SVR was 98\% after treatment of 43 patients with SOF+LED for 3 months. In Fontaine et al. [11], SVR is $100 \%$ in comparison to our study SVR is $98 \%$. We can notice that SVR in our study is less than Fontaine et al. trial [11], and it could be due to, the large number in our study (50 patients in comparison to 15 patients in Fontaine et al. [11], trial, and our study included Naïve patients group, while Fontaine et al. [11], trial included only experienced patients.

In conclusion: Both regimens are effective, well tolerated and associated with high rate of sustained virological response (SVR).

Ethical considrations: This paper was conducted after IRB approval from Al -Azhar university, informed concent was taken from each patient.

Funding: None.

\section{Conflict of interest:None.}

\section{REFERENCES}

1. Mohamed MK. Epidemiology of $\mathrm{HCV}$ in Egypt. The Afro -Arab Liver Journal 2005; $3(2): 41-52$.

2. Kandeel A, Genedy M, El-Refai S, Funk AL, Fontanet A, Talaat $M$. The prevalence of hepatitis C virus infection in Egypt 2015: implications for future policy on prevention and treatment. Liver Int. 2017 Jan;37(1):45-53.

3. van der Meer AJ, Veldt BJ, Feld JJ, Wedemeyer H, Dufour JF, Lammert F, et al. Association between sustained virological response and allcause mortality among patients with chronic hepatitis $\mathrm{C}$ and advanced hepatic fibrosis. JAMA 2012; 308:2584-2593.

4. Lawitz E, Poordad FF, Pang PS, Hyland RH, Ding X, Mo H, et al. Sofosbuvir and ledipasvir fixed-dose combination with and without ribavirin in treatment-naive and previously treated patients with genotype 1 hepatitis $\mathrm{C}$ virus infection (LONESTAR): an open-label, randomised, phase 2 trial. Lancet 2014; 383(9916):515-23.

5. Pol S, Corouge M, Vallet-Pichard A. Daclatasvir-sofosbuvir combination therapy with or without ribavirin for hepatitis $\mathrm{C}$ virus infection: from the clinical trials to real life. Hepatic Medicine: Evidence and Research 2016; 8: 21.

6. Wyles D, Dvory-Sobol H, Svarovskaia ES, Doehle BP, Martin R, Afdhal NH, et al. Posttreatment resistance analysis of hepatitis $\mathrm{C}$ virus from phase II and III clinical trials of ledipasvir/sofosbuvir. J Hepatol 2017; 66:703.

7. Wahid Doss, Magdy El Serrafy, Wafaa el akel , Gamal Esmat, Mohamed Hassany, Mohamed El Kassas, et al. National committee for control of viral hepatitis, National treatment programme of hepatitis $\mathrm{c}$ in Egypt : Hepatitis C virus module of care. 2018.

8. Wong RJ, Jain MK, Therapondos G, Shiffman ML, Kshirsagar O, Clark C, Thamer M.Sustained Improvements in Markers of Liver Disease Severity After Hepatitis C Treatment. $J$ Clin Exp Hepatol. 2020 Mar-Apr;10(2):114-123.

9. Shiha G, Esmat G, Hassany M, Soliman R, Elbasiony M, Fouad R, et al. Ledipasvir/sofosbuvir with or without ribavirin for 8 or 12 weeks for the treatment of $\mathrm{HCV}$ genotype 4 infection: results from a randomized phase III study in Egypt. Gut 2019;68:721-728. 721-728.

10. Ahmed OA, Safwat E, Khalifa MO, Elshafie AI, Fouad MHA, Salama MM, Abd-Elsalam S. Sofosbuvir plus daclatasvir in treatment of chronic hepatitis $\mathrm{C}$ genotype 4 infection in a cohort of Egyptian patients: an experiment the size of Egyptian village. Hindawi International Journal of Hepatology ; Volume 2018, Article ID 9616234, 5 pages

11. Fontaine H, Lazarus A, Pol S, Pecriaux C, Bagate F, Sultanik P, et al. Bradyarrhythmias Associated with Sofosbuvir Treatment. N Engl J Med 2015; 373:1886. 